\title{
MRI software for diffusion-perfusion mismatch analysis may impact on patients' selection and clinical outcome
}

\author{
Silvia Pistocchi ${ }^{1} \cdot$ Davide Strambo $^{2} \cdot$ Bruno Bartolini $^{1} \cdot$ Philippe Maeder $^{1} \cdot$ Reto Meuli $^{1} \cdot$ Patrik Michel $^{2} \cdot$ \\ Vincent Dunet ${ }^{1}$ (D)
}

Received: 29 March 2021 / Revised: 18 June 2021 / Accepted: 9 July 2021 / Published online: 5 August 2021

(C) The Author(s) 2021

\begin{abstract} underwent successful EVT in the early and late time window.

Key Points

- Method C detects fewer patients with favourable mismatch profile.

- Method C might underselect more patients with functional independence at 3 months.

- Software used before thrombectomy may influence patients' outcome.
\end{abstract}

Objective Impact of different MR perfusion software on selection and outcome of patients with acute ischemic stroke (AIS) and large vessel occlusion (LVO) treated by endovascular thrombectomy (EVT) is unclear. We aimed at comparing two commercial MRI software, semi-automated with unadjusted (method A) and adjusted mask (method B), and fully automated (method C) in this setting. Methods MRI from 144 consecutive AIS patients with anterior circulation LVO was retrospectively analysed. All diffusion- and perfusion-weighted images (DWI-PWI) were post-processed with the three methods using standard thresholds. Concordance for core and hypoperfusion volumes was assessed with Lin's test. Clinical outcome was compared between groups in patients who

Results Mean core volume was higher and mean hypoperfusion volume was lower in method $\mathrm{C}$ than in methods $\mathrm{A}$ and $\mathrm{B}$. In the early time window, methods $\mathrm{A}$ and $\mathrm{B}$ found fewer patients with a mismatch ratio $\leq 1.2$ than method C (1/67 [1.5\%] vs. $12 / 67$ $[17.9 \%], p=0.0013)$. In the late time window, methods $\mathrm{A}$ and $\mathrm{B}$ found fewer patients with a mismatch ratio $<1.8$ than method $\mathrm{C}$ $(3 / 46[6.5 \%]$ and $2 / 46[4.3 \%]$ vs. $18 / 46$ [39.1\%], $p \leq 0.0002)$. More patients with functional independence at 3 months would not have been treated using method $\mathrm{C}$ versus methods $\mathrm{A}$ and $\mathrm{B}$ in the early $(p=0.0063)$ and late $(p \leq 0.011)$ time window.

Conclusions MRI software for DWI-PWI analysis may influence patients' selection before EVT and clinical outcome.

Keywords Stroke $\cdot$ Thrombectomy $\cdot$ Software $\cdot$ Diffusion $\cdot$ Perfusion

$\begin{array}{ll}\text { Abbreviations } & \\ \text { AIS } & \text { Acute ischemic stroke } \\ \text { ASTRAL } & \text { Acute STroke Registry and Analysis of } \\ & \text { Lausanne } \\ \text { EVT } & \text { Endovascular thrombectomy } \\ \text { ICA } & \text { Internal carotid artery }\end{array}$

Vincent Dunet

Vincent.Dunet@chuv.ch

1 Diagnostic Neuroradiological Unit, Service of Diagnostic and Interventional Radiology, Department of Medical Radiology, Lausanne University Hospital and University of Lausanne, Lausanne, Switzerland

2 Stroke Center, Service of Neurology, Department of Clinical Neurosciences, Lausanne University Hospital and University of Lausanne, Lausanne, Switzerland
IV tPA Intravenous tissue plasminogen activator

LPGH Last proof of good health

LVO Large vessel occlusions

M1 Middle cerebral artery, horizontal segment

M2 Middle cerebral artery, insular segment

mTICI Modified Treatment in Cerebral Ischemia score

NIHSS National Institutes of Health Stroke Scale

PACS Picture Archiving and Communication System

PWI Perfusion-weighted imaging

sICHECASS Symptomatic intracranial haemorrhage according to the criteria of the European Cooperative Acute Stroke Study

SIT-uv Severely ischemic tissue with uncertain viability

TOAST Trial of ORG 10172 in Acute Stroke Treatment 


\section{Introduction}

Endovascular therapy for patients with acute ischemic stroke (AIS) is nowadays supported by class A evidence [1-6]. Beyond $6 \mathrm{~h}$ from last proof of good health (LPGH), AIS patient with large vessel occlusion (LVO) might still benefit from endovascular therapy when they met either a clinicalcore mismatch or core-hypoperfusion mismatch (i.e. mismatch ratio $\geq 1.8)[7,8]$, using severely ischemic tissue with uncertain viability (SIT-uv) imaging (called 'core' in this manuscript) as a base [9]. As a consequence, perfusion CT or MR imaging is now the standard of care for patients presenting with acute stroke [10]. Many stroke centres introduced automated perfusion processing software in their clinical practice in order to save time and to reduce inter-observer variability [11]. However, it has been proven that some tools require improvement in order to be able to reliably differentiate between patients with a credible ischemia-related region of hypoperfusion and those without [12].

Current literature on AIS perfusion imaging is mostly based on $\mathrm{CT}$ imaging. A comprehensive analysis on CT perfusion imaging [13] has shown that there is marked variability in penumbra and infarct prediction among various deconvolution techniques. Xiong et al [14] compared two automated CT perfusion software (RAPID $\AA$ and Olea Sphere ${ }^{\circledR}$ ) for evaluation of AIS patients, showing that core volumes calculated with RAPID ${ }^{\circledR}$ were larger than with Olea Sphere ${ }^{\circledR}$. The only report about MR perfusion software actually available, recently published by Deutschmann et al [15], compared RAPID ${ }^{\circledR}$ and Olea Sphere $®$ software. They reported a small systematic difference between software, in the sense that RAPID ${ }^{\circledR}$ outlines slightly larger $\mathrm{ADC}$ and smaller hypoperfused tissue volumes. In this context, it seems straightforward to suppose that different perfusion software perform differently in AIS patients' classification. To the best of our knowledge, there is no study that compared RAPID ${ }^{\circledR}$ and Carestream ${ }^{\circledR}$ MR perfusion software and none that evaluated the potential impact on patients' outcome.

In the present study, we aimed at evaluating the concordance between these two software for core and hypoperfusion volumes' estimation in AIS patients with anterior circulation LVO. The second endpoint was to assess the potential impact of volumes' discrepancies on patients' classification prior therapy and on patients' functional independence at 3 months.

\section{Materials and methods}

\section{Patient cohort}

All consecutive patients with acute ischemic stroke and occlusion of ICA or MCA (M1 or M2 segment) who underwent an acute MR imaging between May 2018 and June 2020 at our institution were retrospectively extracted from the Acute STroke Registry and Analysis of Lausanne (ASTRAL) $(N=178)$. ASTRAL collects all acute ischemic strokes admitted to the stroke unit and intensive care unit of the Lausanne University Hospital presenting within $24 \mathrm{~h}$ of stroke onset or LPGH [16].

In this cohort, the inclusion criterion was availability of both DWI and PWI raw data $(N=144 / 178,81 \%)$; MRI with movement artefacts was also included to compare performance of the two software in 'real life' setting. There were no additional exclusion criteria.

Clinical and epidemiological data, including occlusion site, sex, age, cardiovascular risk factors, aetiology classified according to the Trial of Org 10172 in Acute Stroke Treatment (TOAST) criteria [17], National Institutes of Health Stroke Scale (NIHSS) and treatment type, including intravenous tissue plasminogen activator (IV tPA) and/or EVT, were prospectively collected and extracted from ASTRAL. Eligibility for endovascular treatment followed current guidelines. In the early time window, the presence of acute stroke symptoms and anterior circulation LVO was a sufficient criterion to undergo an EVT. In the late time window, we used liberal clinical/imaging mismatch criteria, based on the NIHSS and DWI-ASPECTS, as previously reported [18]. Treatment decision was thus made independently of any software result in order to avoid potential software-related selection bias. Primary revascularisation following EVT was assessed with the modified Treatment in Cerebral Ischemia (mTICI) score. A successful revascularisation was defined as mTICI superior or equal to $2 \mathrm{~b}$. Outcome, expressed as NIHSS at 7 days and modified Rankin score (mRS) at 7 days and at 3 months, and symptomatic intracranial haemorrhage according to the criteria of the European Cooperative Acute Stroke Study (sICHECASS) were also extracted from ASTRAL. A favourable outcome was defined as functional independence (i.e. $\mathrm{mRS}=0$ to 2) [19]. The ethics committee for research on humans of the Canton of Vaud approved collection, analysis and publication of data from ASTRAL, without requesting patients' consent.

\section{Imaging acquisition and post-processing}

All MR examinations were performed on a MAGNETOM VIDA $3 T$ system (Siemens Healthcare) in the Emergency Radiology Unit of our institution, according to the standard protocol used in our department for AIS.

MRI was performed with a 64-channel head coil and included the following sequences: sagittal T1-weighted (TR/ TE: $400.0 \mathrm{~ms} / 2.46 \mathrm{~ms}$, slice thickness $3 \mathrm{~mm}$, FoV $250 \mathrm{~mm}$ ), axial diffusion imaging (TR/TE: $3000.0 \mathrm{~ms} / 80.00 \mathrm{~ms}$, slice thickness $3 \mathrm{~mm}$, FoV $239 \mathrm{~mm}$ ), axial fluid-attenuated inversion recovery (TR/TE: $9000.0 \mathrm{~ms} / 87.00 \mathrm{~ms}$, slice thickness 3 $\mathrm{mm}$, FoV $230 \mathrm{~mm}$ ), axial T2*-weighted gradient echo (TR/ TE: $1070.0 \mathrm{~ms} / 19.80 \mathrm{~ms}$, slice thickness $3 \mathrm{~mm}$, FoV 230 
$\mathrm{mm}$ ), axial 3D TOF angiography (TR/TE: $21.0 \mathrm{~ms} / 3.69 \mathrm{~ms}$, slice thickness $0.5 \mathrm{~mm}$, FoV $220 \mathrm{~mm}$ ), DSC perfusion axial imaging (TE/TR: $2430.0 \mathrm{~ms} / 25.00 \mathrm{~ms}$, slice thickness $3 \mathrm{~mm}$, FoV $215 \mathrm{~mm}$ ) and axial T1-weighted post-contrast media (TE/TR: $400.0 \mathrm{~ms} / 2.61 \mathrm{~ms}$, slice thickness $3 \mathrm{~mm}$, FoV 230 $\mathrm{mm}$ ). Gadolinium-based contrast media (Dotarem, Guerbet) are administered intravenously via a power injection with a dose of $0.1 \mathrm{mmol} / \mathrm{kg}$ body weight at $4 \mathrm{~mL} / \mathrm{s}$ flow followed by $20 \mathrm{~mL}$ of $\mathrm{NaCl} 0.9 \%$ at the same flow, as recommended by ASFNR guidelines [20].

DWI and PWI were processed with two commercial software in three different methods. In all cases, the same thresholds were applied. The core was defined as an apparent diffusion coefficient (ADC) value inferior to $620 \times 10^{-6} \mathrm{~mm}^{2} / \mathrm{s}$ [21] and the hypoperfusion as a $T_{\max }$ superior to $6 \mathrm{~s}[22,23]$. The mismatch ratio was calculated as hypoperfusion/core.

\section{Method A}

Carestream ${ }^{\circledR}$ Picture Archiving and Communication System (PACS) (Version 12.2.2.1025, Philips Healthcare Information Solutions) incorporates stroke perfusion software analysis. Once DWI-PWI raw data arrive on the PACS, the radiologist activates the reconstruction using the 'Perfusion Stroke' module and then chooses the affected hemisphere. No further manipulations of the brain mask are applied for this analysis. This version provides perfusion parameter maps (cerebral blood flow, cerebral blood volume, mean transit time and $T_{\max }$ ) and ADC map. Volumes of core, and hypoperfusion, are calculated in millilitres using thresholds set as previously described, and saved in a table on the PACS. The mismatch ratio (hypoperfusion/core) is manually calculated.

\section{Method B}

Same steps described in method A are followed with additional manual adjustment of the brain mask, which allows suppression of extracerebral mask errors (i.e. suppression of area outside the brain volume) when necessary and is part of standard options of the 'Perfusion Stroke' tool. Core and hypoperfusion volumes are then estimated the same way as described for method A. For the purpose of this study, two neuroradiologists (S.P.

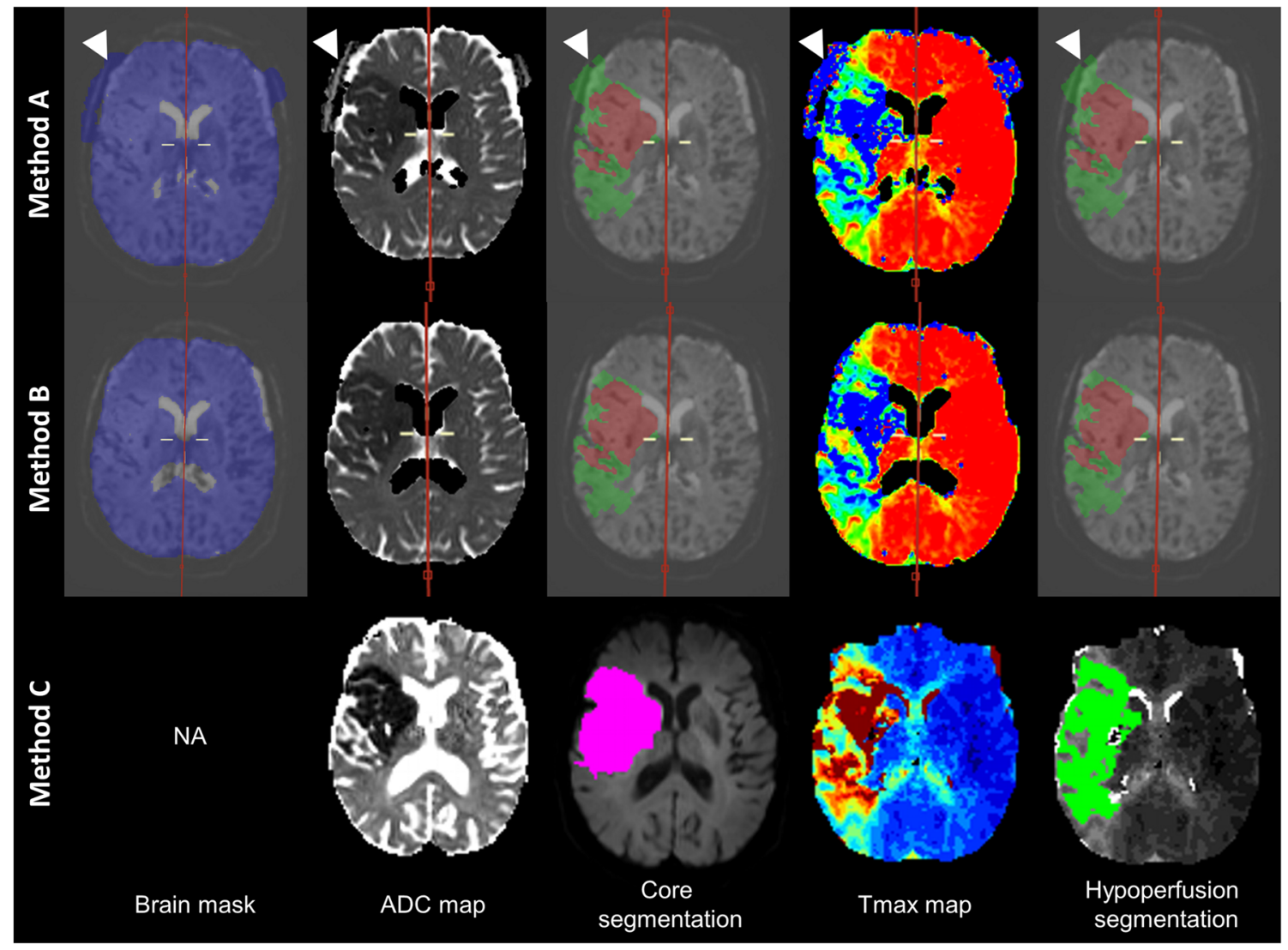

Fig. 1 Exemplary case of volumes' segmentation with the three methods. Case of a 77-year-old female with right M1 segment occlusion diagnosed in the early time window. The core volume, hypoperfusion volume and mismatch ratio were estimated as $47 \mathrm{~mL}, 70 \mathrm{~mL}$ and 1.5 (i.e. favourable mismatch profile) with method A (top line); $47 \mathrm{~mL}, 65 \mathrm{~mL}$ and 1.4 (i.e. favourable mismatch profile) with method B (midline); and $93 \mathrm{~mL}, 100$ $\mathrm{mL}$ and 1.1 (i.e. unfavourable mismatch profile) with method $\mathrm{C}$ (bottom line). White arrows indicate brain mask error found on method A that was removed on method $\mathrm{B}$. The patient underwent an early EVT and had moderate disability at 3 months $(\mathrm{mRS}=3)$ 


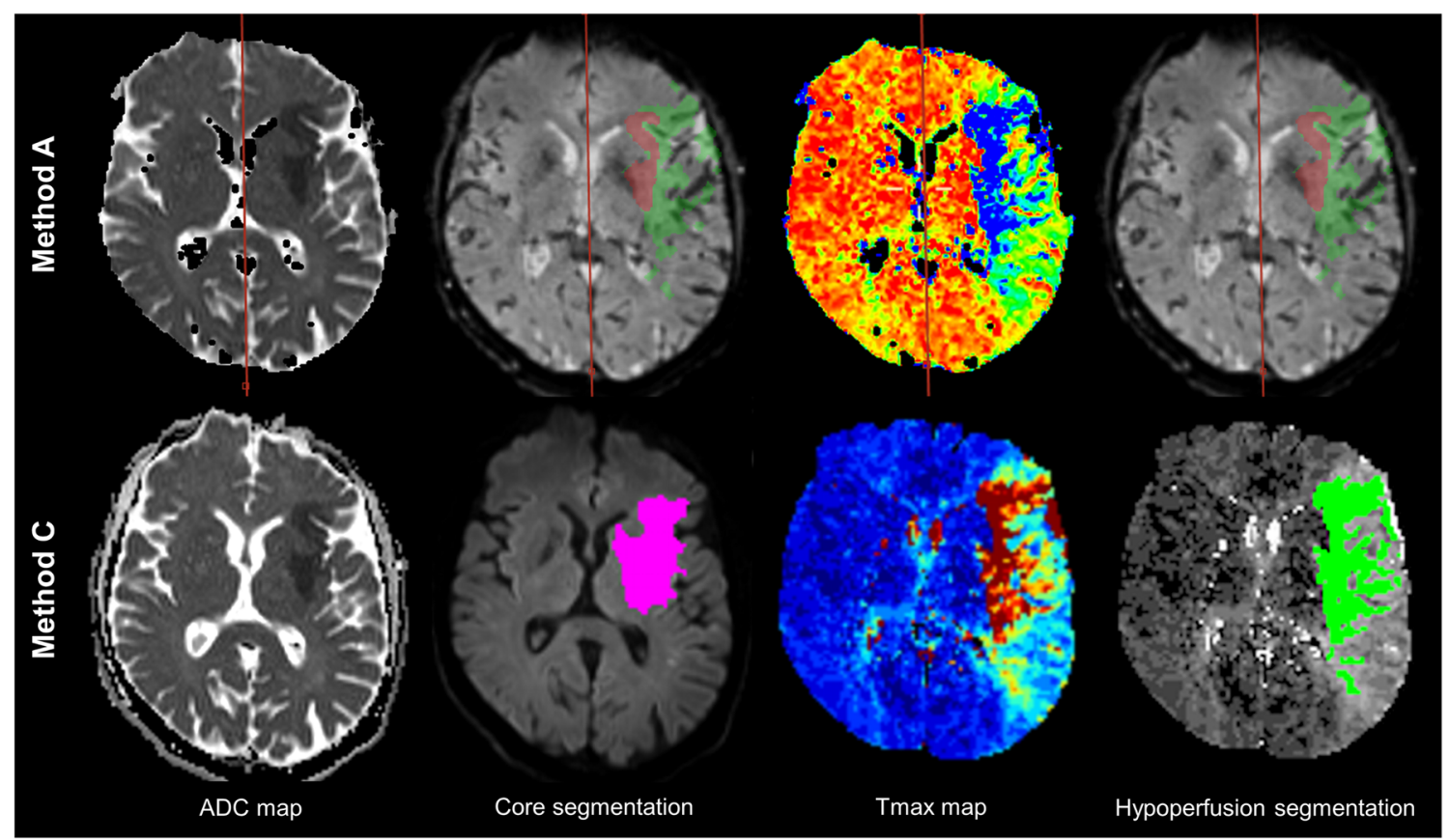

Fig. 2 Exemplary case of volumes' segmentation. Case of a 59-year-old man with left M2 segment occlusion diagnosed in the late time window. The core volume, hypoperfusion volume and mismatch ratio were estimated as $15.2 \mathrm{~mL}, 66.4 \mathrm{~mL}$ and 4.4 (i.e. favourable mismatch profile) with methods A (top line) and $\mathrm{B}$, and $38 \mathrm{~mL}, 65 \mathrm{~mL}$ and 1.7 (i.e. unfavourable mismatch profile) with method $\mathrm{C}$ (bottom line). The patient underwent a late EVT and had functional independence at 3 months $(\mathrm{mRS}=2)$ and V.D. with 10 years' experience in neuroradiology) performed the manual brain mask adjustment of the cohort in consensus reading. This procedure has been performed for all cases and took two additional minutes per case. An exemplary case is shown in Fig. 1.

\section{Method C}

RAPID® (iSchemaView) fully automatically analysed raw data received from MR system and sends the results to our PACS, reporting core and hypoperfusion volumes in millilitres as well as mismatch ratio on a single summary image.

For every method, the core and hypoperfusion volumes were recorded. The DWI-PWI mismatch ratio was recorded in a dichotomous way. In the early time window, a mismatch ratio $>1.2$ defined a favourable profile, while a mismatch ratio $\leq 1.2$ defined an unfavourable profile, according to the FRAME analysis $[3,24]$. In the late time window, a mismatch ratio $\geq 1.8$ defined a favourable profile, while a ratio $<1.8$ defined an unfavourable profile, according to DEFUSE 3 study criteria. An exemplary case is shown in Fig. 2.

\section{Statistical analysis}

All statistics were performed with the Stata software (version 15.0, StataCorp). Continuous variables are presented as mean \pm standard deviation and categorical variables as number or percentage. For further analysis, we divided our study population according to the time window from LPGH to imaging (early $\leq 6 \mathrm{~h}$ or late $>6$ h). Continuous variables and categorical variables were compared between groups using the Wilcoxon signedrank test or Fisher exact test. Inter-method concordance for core and hypoperfusion volume estimation was assessed by Lin's test with estimation of the Pearson correlation coefficient (rho), concordance correlation coefficient (rho_c), bias correction factor $(\mathrm{C}$ b) and calculation of mean difference with $95 \%$ Bland-Altman limits-of-agreement (95\% LOA). Agreement for mismatch-based patients' classification was assessed with Gwet's AC1 $(95 \% \mathrm{CI})$. Correlation coefficient and Gwet's AC1 were interpretated according to the modified Landis and Koch scale as follows: poor when < 0.00 , slight between 0.00 and 0.20 , fair between 0.21 and 0.40 , moderate between 0.41 and 0.60 , good between 0.61 and 0.80 and excellent above 0.81 . Finally, for each method, clinical outcome (NIHSS at 7 days, mRS at 7 days and 3 months) was compared between classification groups in patients who underwent EVT in the early and late windows. A $p$ value $<0.05$ was considered statistically significant. Adjustment of the significance level was done according to the Bonferroni method for every multiple comparisons presented in Tables 4 and 5. 
Table 1 Population characteristics

\begin{tabular}{ll}
\hline Enrolled patients & $N=144$ \\
\hline Males & $71(49.3 \%)$ \\
Age (y) & $74.6 \pm 11.8$ \\
Cardiovascular risk factors & \\
Hypertension & $112(77.7 \%)$ \\
Diabetes & $37(25.7 \%)$ \\
Hypercholesterolemia & $114(79.2 \%)$ \\
Smoking & $38(26.4 \%)$ \\
Atrial fibrillation & $58(40.3 \%)$ \\
Coronaropathy & $36(25 \%)$ \\
Active cancer & $16(11.1 \%)$ \\
Stroke aetiology (TOAST) & \\
Large artery atherosclerosis & $22(15.3 \%)$ \\
Cardiac embolism & $65(45.1 \%)$ \\
Small vessel disease & $0(0 \%)$ \\
Cervical artery dissection & $3(2 \%)$ \\
Patent foramen ovale & $0(0 \%)$ \\
Other determined aetiologies & $10(6.9 \%)$ \\
More than one possible aetiology & $10(6.9 \%)$ \\
Unknown aetiology despite complete evaluation & $22(15.3 \%)$ \\
Unknown aetiology with incomplete evaluation & $6(4.2 \%)$ \\
NIHSS at admission & $12.4 \pm 7.2$ \\
Treatment & \\
IVT & $74(51.4 \%)$ \\
EVT & $115(79.9 \%)$ \\
Conservative & $15(10.4 \%)$ \\
Outcome & \\
NIHSS at 7 days & $9.7 \pm 13.3$ \\
mRS at 7 days & $3.1 \pm 1.6$ \\
mRS 3 months & $3.2 \pm 2.0$ \\
sICHECASS & $5(3.5 \%)$ \\
\hline
\end{tabular}

\section{Results}

\section{Study population and technical success of perfusion imaging}

Demographic data and clinical information for the 144 enrolled patients are provided in Table 1. Out of 144, 74 (74/ $144,51.4 \%)$ were treated with intravenous thrombolysis, 113 $(113 / 144,78.4 \%)$ with EVT and $60(60 / 144,41.7 \%)$ with both in 'bridging' technique. Of 113 patients undergoing EVT, 67 were treated in the early time window and 46 patients in the late time window.

DWI-PWI analysis was successfully performed with methods A and B in all 144 patients and in 142/144 patients (99\%) with method C. In two cases, method C failed to perform the post-processing: in the first case, it did not calculate
Table 2 Estimated volumes and patients' classification

\begin{tabular}{llll}
\hline Parameters & Method A & Method B & Method C \\
\hline $\begin{array}{l}\text { All patients }(N=142) \\
\text { Core }(\mathrm{mL})\end{array}$ & $22.7 \pm 38.7$ & $19.1 \pm 35.3$ & $36.8 \pm 52.2$ \\
Hypoperfusion $(\mathrm{mL})$ & $160.3 \pm 118.9$ & $117.9 \pm 78.4$ & $84.5 \pm 74.0$ \\
Early EVT $(N=67)$ & & & \\
Core $(\mathrm{mL})$ & $19.2 \pm 34.7$ & $15.8 \pm 31.5$ & $31.0 \pm 46.1$ \\
Hypoperfusion $(\mathrm{mL})$ & $166.8 \pm 122.7$ & $121.2 \pm 71.1$ & $82.9 \pm 60.1$ \\
Mismatch ratio & & & \\
$\leq 1.2$ & $1(1.5 \%)$ & $1(1.5 \%)$ & $12(17.9 \%)$ \\
$>1.2$ & $66(98.5 \%)$ & $66(98.5 \%)$ & $55(82.1 \%)$ \\
Late EVT $(N=46)$ & & & \\
Core (mL) & $20.4 \pm 25.7$ & $17.4 \pm 24.4$ & $35.6 \pm 44.5$ \\
Hypoperfusion $(\mathrm{mL})$ & $144.1 \pm 106.4$ & $111.1 \pm 78.6$ & $83.3 \pm 77.8$ \\
Mismatch ratio & & & \\
$<1.8$ & $3(6.5 \%)$ & $2(4.3 \%)$ & $18(39.1 \%)$ \\
$\geq 1.8$ & $43(93.5 \%)$ & $44(95.7 \%)$ & $28(60.9 \%)$ \\
\hline
\end{tabular}

Mismatch ratio cutoff values followed EXTEND-IA criteria in the early time window and DEFUSE 3 criteria in the late time window. EVT endovascular thrombectomy

both core and hypoperfusion volumes, and in the second case, it did not calculate hypoperfusion volume. We excluded these two patients from further analysis.

\section{Inter-method concordance analysis}

Estimated absolute mean values of core and hypoperfusion volumes as well as patients' classification based on dichotomised mismatch ratio are shown in Table 2.

Statistical agreement analysis is shown in Table 3. Regarding core volume, methods $\mathrm{A}$ and $\mathrm{B}$ had an excellent concordance. Compared to method A or B, the core volume was larger using method $\mathrm{C}$ despite an overall good concordance between methods. Regarding hypoperfusion volume, methods $\mathrm{A}$ and $\mathrm{B}$ had a good concordance. Compared to methods A and B, the hypoperfusion volume was lower using method $\mathrm{C}$, with a fair to moderate concordance.

\section{Estimation of unfavourable mismatch profile and outcome in patients who underwent EVT}

\section{Early time window}

In the early time window, of the 67 patients who underwent EVT, 63 (94\%) had successful recanalisation $(\mathrm{mTICI}=2 \mathrm{~b}-3)$. Methods $\mathrm{A}$ and $\mathrm{B}$ found a mismatch ratio $\leq 1.2$ in $1 / 67$ (1.5\%), and method $\mathrm{C}$ in $12 / 67$ (17.9\%) patients. Method $\mathrm{C}$ thus indicated more unfavourable 
Table 3 Inter-method volumes concordance

\begin{tabular}{|c|c|c|c|c|c|}
\hline Pairs & Pearson' rho & Rho_c & $\mathrm{C} \_\mathrm{b}$ & Mean difference & $95 \% \mathrm{LOA}$ \\
\hline \multicolumn{6}{|l|}{ All patients $(N=142)$} \\
\hline \multicolumn{6}{|l|}{ Core volume } \\
\hline Method A versus B & 0.97 & 0.96 & 0.99 & $3.6 \pm 10.0$ & -16.0 to 23.1 \\
\hline Method A versus $C$ & 0.90 & 0.82 & 0.91 & $-14.1 \pm 24.5$ & -62.2 to 34.0 \\
\hline Method B versus $C$ & 0.94 & 0.80 & 0.86 & $-18.1 \pm 23.7$ & -64.5 to 28.2 \\
\hline \multicolumn{6}{|c|}{ Hypoperfusion volume } \\
\hline Method A versus B & 0.74 & 0.62 & 0.85 & $41.8 \pm 80.5$ & -115.9 to 199.6 \\
\hline Method A versus C & 0.38 & 0.27 & 0.69 & $75.8 \pm 113.4$ & -146.5 to 298.2 \\
\hline Method B versus C & 0.56 & 0.51 & 0.91 & $33.4 \pm 71.7$ & -107.1 to 174.0 \\
\hline \multicolumn{6}{|l|}{ Early EVT $(N=67)$} \\
\hline \multicolumn{6}{|l|}{ Core volume } \\
\hline Method A versus B & 0.97 & 0.96 & 0.99 & $3.4 \pm 8.9$ & -14.2 to 20.9 \\
\hline Method A versus C & 0.88 & 0.81 & 0.92 & $-11.8 \pm 22.9$ & -56.8 to 33.1 \\
\hline Method B versus C & 0.92 & 0.79 & 0.87 & $-15.2 \pm 21.5$ & -57.2 to 26.8 \\
\hline \multicolumn{6}{|c|}{ Hypoperfusion volume } \\
\hline Method A versus B & 0.69 & 0.54 & 0.79 & $45.6 \pm 89.7$ & -130.2 to 221.4 \\
\hline Method A versus C & 0.37 & 0.21 & 0.57 & $83.8 \pm 115.0$ & -141.5 to 309.2 \\
\hline Method B versus C & 0.59 & 0.50 & 0.84 & $38.2 \pm 60.0$ & -79.4 to 155.9 \\
\hline \multicolumn{6}{|l|}{ Late $\operatorname{EVT}(N=46)$} \\
\hline \multicolumn{6}{|l|}{ Core volume } \\
\hline Method A versus B & 0.97 & 0.96 & 0.99 & $3.0 \pm 6.5$ & -9.8 to 15.8 \\
\hline Method A versus $\mathrm{C}$ & 0.93 & 0.74 & 0.80 & $-15.2 \pm 22.5$ & -59.3 to 28.8 \\
\hline Method B versus C & 0.94 & 0.70 & 0.75 & $-18.2 \pm 23.2$ & -63.7 to 27.2 \\
\hline \multicolumn{6}{|c|}{ Hypoperfusion volume } \\
\hline Method A versus B & 0.88 & 0.79 & 0.90 & $33.0 \pm 52.4$ & -69.6 to 135.7 \\
\hline Method A versus C & 0.51 & 0.40 & 0.78 & $60.8 \pm 94.6$ & -124.6 to 246.2 \\
\hline Method B versus C & 0.63 & 0.60 & 0.94 & $27.8 \pm 67.0$ & -103.5 to 159.0 \\
\hline
\end{tabular}

$E V T$ endovascular thrombectomy, $L O A$ limits-of-agreement mismatch profile $(p=0.0013)$. The agreement to identify a mismatch ratio $\leq 1.2$ vs. $>1.2$ was excellent between methods A and B (Gwet's AC1: 0.97 [0.92-1.0]) and only good between method C and methods A and B (Gwet's $\mathrm{AC} 1: 0.76$ [0.62-0.90]). Regarding patients' outcome for these two subgroups, only one patient was classified as having an unfavourable mismatch profile with methods A and $\mathrm{B}$, and had a poor outcome despite successful recanalization. All of the 31/63 (49.2\%) patients who were functionally independent at 7 days and 3 months after successful EVT had a favourable mismatch profile according to methods A and B. Compared to patients with an unfavourable mismatch profile, the proportion of patients with a favourable mRS at 7 days and 3 months was higher in the case of a favourable mismatch profile as defined by methods A, B and C. However, 6/31 (19.4\%) patients with a favourable outcome would not have been treated when only taking into account the mismatch profile attributed by method $\mathrm{C}$ (versus $0 \%$ with methods $\mathrm{A}$ and $\mathrm{B}, p=0.0063$ ). All outcome data are reported in Table 4.

\section{Late time window}

In the late time window, of the 46 patients who underwent EVT, 42 (91.3\%) had successful recanalization. Method A indicated a mismatch ratio $<1.8$ in $3 / 46(6.5 \%)$ patients, method B in 2/46 (4.3\%) patients and method C in 18/46 (39.1\%) patients $(p \leq 0.0002)$. The agreement was excellent between methods A and B (Gwet's AC1: 0.93 [0.84-1.0]), but it was only moderate between method $\mathrm{C}$ and methods $\mathrm{A}$ and $\mathrm{B}$ (Gwet's AC1: 0.50 [0.22-0.77] and 0.47 [0.20-0.75], respectively). Compared to patients with an unfavourable mismatch profile, the proportion of patients with a favourable mRS at 7 days and 3 months was higher in the case of a favourable mismatch profile as defined by methods A and $\mathrm{B}$ ( $p \leq$ 0.0002). On the contrary, the proportion of patients with a favourable mRS at 7 days and 3 months was similar between patients with an unfavourable and a favourable mismatch profile defined by method C ( $p \geq 0.014)$. Out of 18 patients having a good mRS at 3 months, 7 (38.9\%) would not have been treated taking into account the profile attributed by 
Table 4 EVT patients' outcome in the early time window

\begin{tabular}{|c|c|c|c|c|}
\hline Method A & $\mathrm{TICI} 2 \mathrm{~b} / 2 \mathrm{c} / 3, n=63$ & $\mathrm{MR} \leq 1.2, n=1$ & $\mathrm{MR}>1.2, n=62$ & $p$ value \\
\hline NIHSS admission & $12.3 \pm 7.3$ & 27 & $12.1 \pm 7.1$ & NA \\
\hline NIHSS 7d & $7.4 \pm 12.2^{\ddagger}$ & 28 & $7.0 \pm 12.0^{\ddagger}$ & NA \\
\hline $\mathrm{mRS}$ pre-stroke & $1.0 \pm 1.1$ & 0 & $1.1 \pm 1.1$ & NA \\
\hline $\mathrm{mRS} 7 \mathrm{~d}$ & $2.8 \pm 1.6$ & 5 & $2.7 \pm 1.6$ & NA \\
\hline mRS 3 months & $2.7 \pm 1.9$ & 3 & $2.7 \pm 1.9$ & NA \\
\hline $\mathrm{mRS} 0-2$ at $7 \mathrm{~d}$ & $31 / 63(49.2 \%)$ & $0 / 63(0 \%)$ & $31 / 63(49.2 \%)$ & $<0.0001$ \\
\hline $\mathrm{mRS} 0-2$ at $3 \mathrm{~m}$ & $31 / 63(49.2 \%)$ & $0 / 63(0 \%)$ & $31 / 63(49.2 \%)$ & $<0.0001$ \\
\hline sICHECASS & $3 / 63(4.8 \%)$ & $0 / 63(0 \%)$ & $3 / 63(4.8 \%)$ & 0.07 \\
\hline Method B & $\mathrm{TICI} 2 \mathrm{~b} / 2 \mathrm{c} / 3, n=63$ & $\mathrm{MR} \leq 1.2, n=1$ & $\mathrm{MR}>1.2, n=62$ & $p$ value \\
\hline NIHSS admission & $12.3 \pm 7.3$ & 20 & $12.2 \pm 7.3$ & NA \\
\hline NIHSS 7d & $7.4 \pm 12.2^{\ddagger}$ & 42 & $6.8 \pm 11.4^{*}$ & NA \\
\hline mRS pre-stroke & $1.0 \pm 1.1$ & 0 & $1.1 \pm 1.1$ & NA \\
\hline $\operatorname{mRS} 7 d$ & $2.8 \pm 1.6$ & 6 & $2.7 \pm 1.5$ & NA \\
\hline mRS 3 months & $2.7 \pm 1.9$ & 6 & $2.6 \pm 1.9$ & NA \\
\hline $\mathrm{mRS} 0-2$ at $7 \mathrm{~d}$ & $31 / 63(49.2 \%)$ & $0 / 63(0 \%)$ & $31 / 63(49.2 \%)$ & $<0.0001$ \\
\hline $\mathrm{mRS} 0-2$ at $3 \mathrm{~m}$ & $31 / 63(49.2 \%)$ & $0 / 63(0 \%)$ & $31 / 63(49.2 \%)$ & $<0.0001$ \\
\hline sICHECASS & $3 / 63(4.8 \%)$ & $0 / 63(0 \%)$ & $3 / 63(4.8 \%)$ & 0.07 \\
\hline Method C & $\mathrm{TICI} 2 \mathrm{~b} / 2 \mathrm{c} / 3, n=63$ & $\mathrm{MR} \leq 1.2, n=12$ & $\mathrm{MR}>1.2, n=51$ & $p$ value \\
\hline NIHSS admission & $12.3 \pm 7.3$ & $13.2 \pm 8.0$ & $12.1 \pm 7.1$ & 0.62 \\
\hline NIHSS 7d & $7.4 \pm 12.2^{\ddagger}$ & $6.6 \pm 7.7$ & $7.6 \pm 13.2^{\dagger}$ & 0.48 \\
\hline mRS pre-stroke & $1.0 \pm 1.1$ & $1.0 \pm 0.9$ & $1.1 \pm 1.2$ & 0.98 \\
\hline $\operatorname{mRS} 7 d$ & $2.8 \pm 1.6$ & $2.8 \pm 1.6$ & $2.8 \pm 1.6$ & 0.21 \\
\hline mRS 3 months & $2.7 \pm 1.9$ & $2.6 \pm 2.1$ & $2.7 \pm 1.9$ & 0.23 \\
\hline $\mathrm{mRS} 0-2$ at $7 \mathrm{~d}$ & $31 / 63(49.2 \%)$ & $6 / 63(9.5 \%)$ & $25 / 63(39.7 \%)$ & $<0.0001$ \\
\hline $\mathrm{mRS} 0-2$ at $3 \mathrm{~m}$ & $31 / 63(49.2 \%)$ & $6 / 63(9.5 \%)$ & $25 / 63(39.7 \%)$ & $<0.0001$ \\
\hline sICHECASS & $3 / 63(4.8 \%)$ & $0 / 63(0 \%)$ & $3 / 63(4.8 \%)$ & 0.07 \\
\hline
\end{tabular}

EVT endovascular thrombectomy, $M R$ mismatch ratio, $m R S$ modified Rankin scale, NIHSS National Institutes of Health Stroke Scale, TICI Thrombolysis in Cerebral Infarction. $p$ values in the right column referred to comparison between favourable and unfavourable mismatch profiles. $p$ value $<0.05$, corr $p$ value $<0.005 .{ }^{\dagger} p<0.05$ for NIHSS at 7 days compared to NIHSS at admission. ${ }^{\ddagger} p<0.005$ for NIHSS at 7 days compared to NIHSS at admission method $\mathrm{C}$ versus $3(16.6 \%)$ and $1(5.5 \%)$ using methods A and $\mathrm{B}(p=0.011$ and $p<0.0001$, respectively). All outcome data are reported in Table 5.

\section{Discussion}

Three main results from the present study are as follows: (1) despite using identical core/penumbra thresholds, core volumes are larger and hypoperfusion volumes are smaller when estimated by method C; (2) this results in a higher proportion of patients with unfavourable DWI-PWI mismatch profile using method $\mathrm{C}$, both in the early and late time windows; (3) in patients with functional independence at 3 months after successful late EVT, method C would have underselected more patients than methods A and B. Overall the analysis of outcome of patients that underwent successful EVT suggests that some DWI-PWI software may be too restrictive.

MRI is considered the gold standard in the detection of AIS. Nevertheless, unlike CT, MRI is not widely available in acute stroke settings. Accordingly, current literature on AIS perfusion imaging based on MR imaging modality is still scarce.

In this study, we compared two commercially available MR perfusion software using three methods. We found larger core volume and lower hypoperfusion volume using method $\mathrm{C}$ compared to methods $\mathrm{A}$ and $\mathrm{B}$. This observation was made for AIS patients with anterior circulation LVO admitted either in the early or in late time window who underwent EVT. This is in line with the study by Deutschmann et al [15] who 
Table 5 EVT patients' outcome in the late time window

\begin{tabular}{|c|c|c|c|c|}
\hline Method A & TICI2b/3, $n=42$ & $\mathrm{MR}<1.8, n=3$ & $\mathrm{MR} \geq 1.8, n=39$ & $p$ value \\
\hline NIHSS admission & $13.8 \pm 6.3$ & $9.3 \pm 3.1$ & $14.2 \pm 6.4$ & 0.14 \\
\hline NIHSS 7d & $8.6 \pm 11.0^{\dagger}$ & $3.0 \pm 2.6^{\dagger}$ & $9.1 \pm 11.4^{\dagger}$ & 0.58 \\
\hline $\mathrm{mRS}$ pre-stroke & $1.1 \pm 1.1$ & $1.3 \pm 0.6$ & $1.1 \pm 1.1$ & 0.54 \\
\hline mRS 7d & $3.0 \pm 1.4$ & $2.3 \pm 0.6$ & $3.1 \pm 1.5$ & 0.36 \\
\hline mRS 3 months & $3.0 \pm 1.9$ & $1.7 \pm 0.6$ & $3.1 \pm 1.9$ & 0.21 \\
\hline $\operatorname{mRS} 0-2$ at $7 \mathrm{~d}$ & $15 / 42(35.7 \%)$ & $2 / 42(4.8 \%)$ & $13 / 42(31.0 \%)$ & 0.0002 \\
\hline $\mathrm{mRS} 0-2$ at $3 \mathrm{~m}$ & $18 / 42(42.9 \%)$ & $3 / 42(7.1 \%)$ & $15 / 42(35.7 \%)$ & 0.0001 \\
\hline sICHECASS & $2 / 42(4.8 \%)$ & $0 / 42(0 \%)$ & $2 / 42(4.8 \%)$ & 0.27 \\
\hline Method B & $\mathrm{TICI} 2 \mathrm{~b} / 3, n=42$ & $\mathrm{MR}<1.8, n=2$ & $\mathrm{MR} \geq 1.8, n=40$ & $p$ value \\
\hline NIHSS admission & $13.8 \pm 6.3$ & $11.0 \pm 1.4$ & $14.0 \pm 6.4$ & 0.46 \\
\hline NIHSS 7d & $8.6 \pm 11.0^{\dagger}$ & $18.5 \pm 24.7$ & $8.1 \pm 10.2^{\ddagger}$ & 0.57 \\
\hline mRS pre-stroke & $1.1 \pm 1.1$ & $1.0 \pm 0.0$ & $1.2 \pm 1.1$ & 1.0 \\
\hline $\operatorname{mRS} 7 d$ & $3.0 \pm 1.4$ & $3.5 \pm 2.1$ & $3.0 \pm 1.4$ & 0.63 \\
\hline mRS 3 months & $3.0 \pm 1.9$ & $3.5 \pm 3.5$ & $2.9 \pm 1.8$ & 0.94 \\
\hline $\operatorname{mRS} 0-2$ at $7 \mathrm{~d}$ & $15 / 42(35.7 \%)$ & $1 / 42(2.4 \%)$ & $14 / 42(33.3 \%)$ & $<0.0001$ \\
\hline $\mathrm{mRS} 0-2$ at $3 \mathrm{~m}$ & $18 / 42(42.9 \%)$ & $1 / 42(2.4 \%)$ & $17 / 42(40.5 \%)$ & $<0.0001$ \\
\hline sICHECASS & $2 / 42(4.8 \%)$ & $0 / 42(0 \%)$ & $2 / 42(4.8 \%)$ & 0.27 \\
\hline Method C & TICI2b/3, $n=42$ & $\mathrm{MR}<1.8, n=16$ & $\mathrm{MR} \geq 1.8, n=26$ & $p$ value \\
\hline NIHSS admission & $13.8 \pm 6.3$ & $14.9 \pm 6.1$ & $13.2 \pm 6.4$ & 0.53 \\
\hline NIHSS 7d & $8.6 \pm 11.0^{\dagger}$ & $9.3 \pm 9.8^{\dagger}$ & $8.1 \pm 12.1^{\dagger}$ & 0.28 \\
\hline mRS pre-stroke & $1.1 \pm 1.1$ & $1.3 \pm 0.8$ & $1.0 \pm 1.2$ & 0.18 \\
\hline $\operatorname{mRS} 7 \mathrm{~d}$ & $3.0 \pm 1.4$ & $3.2 \pm 1.1$ & $2.9 \pm 1.6$ & 0.46 \\
\hline mRS 3 months & $3.0 \pm 1.9$ & $2.9 \pm 1.7$ & $3.0 \pm 2.1$ & 0.88 \\
\hline $\operatorname{mRS} 0-2$ at $7 \mathrm{~d}$ & $15 / 42(35.7 \%)$ & $4 / 42(9.5 \%)$ & $11 / 42(26.2 \%)$ & 0.014 \\
\hline $\mathrm{mRS} 0-2$ at $3 \mathrm{~m}$ & $18 / 42(42.9 \%)$ & $7 / 42(16.7 \%)$ & $11 / 42(26.2 \%)$ & 0.16 \\
\hline sICHECASS & $2 / 42(4.8 \%)$ & $1 / 42(2.4 \%)$ & $1 / 42(2.4 \%)$ & 1.0 \\
\hline
\end{tabular}

$E V T$ endovascular thrombectomy, MR mismatch ratio, $m R S$ modified Rankin scale, NIHSS National Institutes of Health Stroke Scale, TICI Thrombolysis in Cerebral Infarction. $p$ values in the right column referred to comparison between favourable and unfavourable mismatch profiles. $p$ value $<0.05$, corr $p$ value $<0.005 .{ }^{\dagger} p<0.05$ for NIHSS at 7 days compared to NIHSS at admission. ${ }^{\ddagger} p<0.005$ for NIHSS at 7 days compared to NIHSS at admission compared RAPID ${ }^{\circ}$ and Olea Sphere ${ }^{\circledR}$ software and reported that RAPID ${ }^{\circledR}$ outlines slightly larger ADC and smaller hypoperfused tissue volumes. They considered that small differences of the outlined regions in single slices can sum up to considerable differences in 3D volumes. Our results are also in line with those reported by Xiong et al [14, 25]. However, the potential impact on patients' selection and outcome either in the early or in the late time window remained unclear.

According to the American Heart Association [26] guidelines, no specific advanced imaging selection criteria are required for EVT in patients who present within $6 \mathrm{~h}$ of LPGH and have anterior circulation LVO. Nevertheless, there are proofs that perfusion imaging profiles predict the clinical response to EVT even in this early time window $[3,24]$ when applying the criterion of ischemic core $<70 \mathrm{~mL}$ and a DWI-
PWI mismatch ratio $>1.2$ [3]. In accordance, we found that up to $49.2 \%$ of patients having a favourable mismatch profile had functional independence at 3 months after successful EVT. However, our results also indicate that method $\mathrm{C}$ is more restrictive for patients' selection, while $19.3 \%$ of patients with a good outcome would not have undergone EVT when basing selection only on imaging criterion. As all multicentre studies used method C for patients' selection, our results may suggest that more AIS patients could in fact benefit from EVT in the early time window than previously reported. This may also imply that selection for EVT should not be only based on imaging criterion in the early time window.

In the late time window, EVT is recommended for AIS patients who present within 6 to $16 \mathrm{~h}$ of LPGH, have anterior circulation LVO and meet the DEFUSE 3 (infarct core $<70$ 
$\mathrm{mL}$, mismatch ratio $\geq 1.8$ ) or DAWN (mismatch between the severity of the clinical deficit and the infarct volume) inclusion criterion. In the present study, when applying DEFUSE 3 criterion at our cohort, a larger number of patients was classified has having an unfavourable mismatch profile when using method $C$ versus $A$ and $B$. This indicates that method $C$ could be more restrictive for selecting patients eligible for EVT. Indeed, in the late time window, 16/42 (38.1\%) patients with successful EVT would not have undergone EVT with method C compared to $3 / 42(7.1 \%)$ or $2 / 42(4.8 \%)$ with method A or B. This is in line with Deutschmann et al [15] who reported that application of the DEFUSE 3 threshold (i.e. core $<70 \mathrm{~mL}$ only) would have resulted in revoking $7.4 \%$ of patients with RAPID® compared to Olea Sphere ${ }^{\circledR}$. Interestingly, of 18 patients with functional independence at 3 months, $7 / 18$ (38.9\%) would not have been treated using method $\mathrm{C}$, which suggests that patients' outcome may be significantly influenced by the type of software used for initial imaging evaluation. Ducroux et al [27] reported that 7/17 (41\%) DEFUSE 3-ineligible patients and 16/42 (38\%) DAWN-ineligible patients who underwent EVT did benefit from this treatment. This could be partly due to software package as we found that $7 / 16$ $(43.8 \%)$ patients with unfavourable mismatch profile as indicated by method $\mathrm{C}$ had functional independence at 3 months. Similarly, Desai et al [28] reported that $30 \%$ of patients undergoing off-label EVT according to the DAWN and DEFUSE 3 criteria when assessed with method $C$ achieved functional independence at 3 months. This might overall suggest that more AIS patients could benefit from late EVT than reported in multicentre trials using optimised perfusion software because they were underselected.

We have to acknowledge several limitations in our study. First, this is a retrospective study, even if it relies on a prospectively collected and consecutive cohort of patients, which can reduce some selection bias. Second, there is a risk of bias in a monocentric study; its advantage is the uniformity of clinical data collection following an identical protocol at 3T on a single MR scanner. Third, our imaging protocol used high-resolution 3-mm slice thickness diffusion and perfusion imaging, which could limit interpolation-related overestimation due to thicker slices, although the manufacturer of method $\mathrm{C}$ does recommend $5-\mathrm{mm}$ slice thickness acquisition. Moreover, comparing methods $\mathrm{A}$ and $\mathrm{C}$, all manual interferences on volume segmentation were avoided, both methods being independent from user manipulation-related bias and experience. On the contrary, method B included mask quality control and manual adjustment. However, this needed only minimal expertise and was poorly time-consuming ( 2 min per case). Furthermore, we found that revocation of patients with a good outcome might occur at a lower rate in the late time window using method B (5.5\% versus $16.6 \%$ and $38.9 \%$ for methods $\mathrm{A}$ and $\mathrm{C}$ ), which suggests that losing 2 min of time could at the end help saving functional independence in some patients. Taking into account recent development of artificial intelligence-based software, use of machine learning or more advanced deep learning technologies in perfusion tools may help in improving volume delineation, reduce inter-reader and inter-software variability and harmonise patients' triage [29]. This was out of the scope of our work and needs to be evaluated in large prospective multicentre studies.

\section{Conclusion}

MRI software used for DWI-PWI analysis may influence patients' selection before EVT and impact clinical outcome either at the early and late time window. More restrictive software could deprive some AIS patients of the functional benefit of a successful endovascular thrombectomy. Quality control and manual refining should be considered when using fully automated software not to preclude functional independence of some patients.

Funding Open Access funding provided by Université de Lausanne.

\section{Declarations}

Guarantor The scientific guarantor of this publication is Vincent Dunet.

Conflict of interest The authors of this manuscript declare no relationships with any companies whose products or services may be related to the subject matter of the article.

Statistics and biometry One of the authors (Vincent Dunet) has significant statistical expertise. No complex statistical methods were necessary for this paper.

Informed consent Written informed consent was waived by the Institutional Review Board.

Ethical approval Institutional Review Board approval was obtained. The ethics committee for research on humans of the Canton of Vaud approved collection, analysis and publication of data from ASTRAL, without requesting patients' consent.

\section{Methodology \\ - retrospective \\ - observational \\ - performed at one institution}

Open Access This article is licensed under a Creative Commons Attribution 4.0 International License, which permits use, sharing, adaptation, distribution and reproduction in any medium or format, as long as you give appropriate credit to the original author(s) and the source, provide a link to the Creative Commons licence, and indicate if changes were made. The images or other third party material in this article are included in the article's Creative Commons licence, unless indicated otherwise in a credit line to the material. If material is not included in the article's Creative Commons licence and your intended use is not permitted by statutory regulation or exceeds the permitted use, you will need to obtain 
permission directly from the copyright holder. To view a copy of this licence, visit http://creativecommons.org/licenses/by/4.0/.

\section{References}

1. Berkhemer OA, Fransen PSS, Beumer D et al (2015) A randomized trial of intraarterial treatment for acute ischemic stroke. N Engl $\mathrm{J}$ Med 372(1):11-20

2. Bracard S, Ducrocq X, Mas JL et al (2016) Mechanical thrombectomy after intravenous alteplase versus alteplase alone after stroke (THRACE): a randomised controlled trial. Lancet Neurol 15(11):1138-1147

3. Campbell BC, Mitchell PJ, Kleinig TJ et al (2015) Endovascular therapy for ischemic stroke with perfusion-imaging selection. $\mathrm{N}$ Engl J Med 372(11):1009-1018

4. Goyal M, Demchuk AM, Menon BK et al (2015) Randomized assessment of rapid endovascular treatment of ischemic stroke. $\mathrm{N}$ Engl J Med 372(11):1019-1030

5. Jovin TG, Chamorro A, Cobo E et al (2015) Thrombectomy within 8 hours after symptom onset in ischemic stroke. N Engl J Med 372(24):2296-2306

6. Saver JL, Goyal M, Bonaf A et al (2015) Stent-retriever thrombectomy after intravenous t-PA vs. t-PA alone in stroke. N Engl J Med 372(24):2285-2295

7. Albers GW, Marks MP, Kemp S et al (2018) Thrombectomy for stroke at 6 to 16 hours with selection by perfusion imaging. N Engl J Med 378(8):708-718

8. Nogueira RG, Jadhav AP, Haussen DC et al (2018) Thrombectomy 6 to 24 hours after stroke with a mismatch between deficit and infarct. $\mathrm{N}$ Engl J Med 378(1):11-21

9. Goyal $\mathrm{M}$ et al (2020) Challenging the ischemic core concept in acute ischemic stroke imaging. Stroke 51(10):3147-3155

10. Warach SJ et al (2016) Acute stroke imaging research roadmap III imaging selection and outcomes in acute stroke reperfusion clinical trials: consensus recommendations and further research priorities. Stroke 47(5):1389-1398

11. Khoury N, Dargazanli C, Guenego A et al (2019) Visual assessment of diffusion weighted imaging infarct volume lacks accuracy and reliability. J Neurointerv Surg 11(9):947-954

12. Galinovic I, Brunecker P, Ostwaldt AC, Soemmer C, Hotter B, Fiebach JB (2011) Fully automated postprocessing carries a risk of substantial overestimation of perfusion deficits in acute stroke magnetic resonance imaging. Cerebrovasc Dis 31(4):408-413

13. Bivard A, Levi C, Spratt N, Parsons M (2013) Perfusion CT in acute stroke: a comprehensive analysis of infarct and penumbra. Radiology 267(2):543-550

14. Xiong Y, Huang CC, Fisher M, Hackney DB, Bhadelia RA, Selim MH (2019) Comparison of automated CT perfusion softwares in evaluation of acute ischemic stroke. J Stroke Cerebrovasc Dis 28(12): 104392

15. Deutschmann, H, Hinteregger N, Wießpeiner U et al (2021) Automated MRI perfusion-diffusion mismatch estimation may be significantly different in individual patients when using differentsoftware packages. Eur Radio 31(2):658-665
16. Michel P, Odier C, Rutgers M et al (2010) The Acute STroke Registry and Analysis of Lausanne (ASTRAL): design and baseline analysis of an ischemic stroke registry including acute multimodal imaging. Stroke 41(11):2491-2498

17. Adams HP Jr, Bendixen BH, Kappelle LJ et al (1993) Classification of subtype of acute ischemic stroke. Definitions for use in a multicenter clinical trial. TOAST. Trial of Org 10172 in Acute Stroke Treatment. Stroke 24(1):35-41

18. Nannoni S, Strambo D, Sirimarco G et al (2020) Eligibility for late endovascular treatment using DAWN, DEFUSE-3, and more liberal selection criteria in a stroke center. J Neurointerv Surg 12(9): 842-847

19. Menjot de Champfleur N, Saver JL, Goyal M et al (2017) Efficacy of stent-retriever thrombectomy in magnetic resonance imaging versus computed tomographic perfusion-selected patients in SWIFT PRIME Trial (Solitaire FR With the Intention for Thrombectomy as Primary Endovascular Treatment for Acute Ischemic Stroke). Stroke 48(6):1560-1566

20. Welker K, Boxerman J, Kalnin A et al (2015) ASFNR recommendations for clinical performance of MR dynamic susceptibility contrast perfusion imaging of the brain. AJNR Am J Neuroradiol 36(6): E41-E51

21. Purushotham A, Campbell BCV, Straka M et al (2015) Apparent diffusion coefficient threshold for delineation of ischemic core. Int $\mathrm{J}$ Stroke 10(3):348-353

22. Christensen S, Mouridsen K, Wu O et al (2009) Comparison of 10 perfusion MRI parameters in 97 sub-6-hour stroke patients using voxel-based receiver operating characteristics analysis. Stroke 40(6):2055-2061

23. Wouters A, Christensen S, Straka M et al (2017) A comparison of relative time to peak and Tmax for mismatch-based patient selection. Front Neurol 8:539

24. Olivot JM, Albucher JF, Guenego A et al (2021) Mismatch profile influences outcome after mechanical thrombectomy. Stroke 52(1): 232-240

25. Austein F, Riedel C, Kerby T et al (2016) Comparison of perfusion CT software to predict the final infarct volume after thrombectomy. Stroke 47(9):2311-2317

26. Powers WJ, Rabinstein AA, Ackerson T et al (2018) 2018 guidelines for the early management of patients with acute ischemic stroke: a guideline for healthcare professionals from the American Heart Association/American Stroke Association. Stroke 49(3):e46 e110

27. Ducroux C, Khoury N, Lecler A et al (2018) Application of the DAWN clinical imaging mismatch and DEFUSE 3 selection criteria: benefit seems similar but restrictive volume cut-offs might omit potential responders. Eur J Neurol 25(8):1093-1099

28. Desai SM, Rocha M, Molyneaux BJ et al (2018) Thrombectomy 624 hours after stroke in trial ineligible patients. J Neurointerv Surg 10(11):1033-1037

29. Murray NM, Unberath M, Hage GD, Hui FK (2020) Artificial intelligence to diagnose ischemic stroke and identify large vessel occlusions: a systematic review. J Neurointerv Surg 12(2):156-164

Publisher's note Springer Nature remains neutral with regard to jurisdictional claims in published maps and institutional affiliations. 